\title{
The white-nosed coati (Nasua narica) is a naturally susceptible definitive host for the zoonotic nematode Angiostrongylus costaricensis in Costa Rica
}

\author{
Mario Santoro ${ }^{\mathrm{a}, *}$, Alejandro Alfaro-Alarcón ${ }^{\mathrm{b}}$, Vincenzo Veneziano ${ }^{\mathrm{c}}$, Anna Cerrone ${ }^{\mathrm{a}}$, \\ Maria Stefania Latrofa ${ }^{\mathrm{d}}$, Domenico Otranto ${ }^{\mathrm{d}}$, Isabel Hagnauer ${ }^{\mathrm{b}}$, Mauricio Jiménez ${ }^{\mathrm{b}}$, \\ Giorgio Galiero ${ }^{a}$
}

a Istituto Zooprofilattico Sperimentale del Mezzogiorno, Portici, Naples, Italy

bscuela de Medicina Veterinaria, Universidad Nacional, Heredia, Costa Rica

c Department of Veterinary Medicine and Animal Production, University of Naples Federico II, Naples, Italy

${ }^{\mathrm{d}}$ Dipartimento di Medicina Veterinaria, Università degli Studi di Bari, Valenzano, Bari, Italy

\section{A R T I C L E I N F O}

\section{Article history:}

Received 17 June 2016

Received in revised form 23 August 2016

Accepted 24 August 2016

\section{Keywords:}

Abdominal angiostrongyliasis

Procyon lotor

Zoonotic helminth

Parasitic disease

Public health

Reservoir

\begin{abstract}
A B S T R A C T
Angiostrongylus costaricensis (Strongylida, Angiostrongylidae) is a roundworm of rodents, which may cause a severe or fatal zoonosis in several countries of the Americas. A single report indicated that the white-nosed coati (Nasua narica), acts as a potential free-ranging wildlife reservoir. Here we investigated the prevalence and features of $A$. costaricensis infection in two procyonid species, the white-nosed coati and the raccoon (Procyon lotor) from Costa Rica to better understand their possible role in the epidemiology of this zoonotic infection. Eighteen of 32 (56.2\%) white-nosed coatis collected between July 2010 and March 2016 were infected with $A$. costaricensis but none of 97 raccoons from the same localities were diagnosed with this infection. First-stage larvae of $A$. costaricensis were obtained from feces of 17 fresh white-nosed coati carcasses by Baermann technique. Parasite identity was confirmed by morphology, histology and molecular characterization of target genes. These data demonstrate that the white-nosed coati is a naturally susceptible definitive host for A. costaricensis in Costa Rica contrary to findings in the raccoon.
\end{abstract}

(c) 2016 Elsevier B.V. All rights reserved.

\section{Introduction}

Angiostrongylus costaricensis (Strongylida, Angiostrongylidae), a zoonotic nematode causing severe or fatal abdominal angiostrongyliasis in humans, develops in a wide range of rodents (i.e., cotton rat Sigmodon hispidus) which act as definitive hosts (Morera and Céspedes, 1971; Spratt, 2015). In addition, naturally infected white-nosed coati (Nasua narica) and dogs have been suggested to act as reservoir for this parasite (Monge et al., 1978; Alfaro-Alarcón et al., 2016) and they may shed first-stage larvae (L1) in their feces. Angiostrongylus costaricensis was also reported from mesenteric arteries of free-ranging raccoons (Procyon lotor) and an opossum (Didelphis virginiana) in Florida (United States)

\footnotetext{
* Corresponding author.

E-mail addresses: marisant@libero.it, mario.santoro@izsmportici.it (M. Santoro).
}

although, fecal shedding of L1 was not demonstrated (Miller et al., 2006).

Adult worms live in the mesenteric arteries of the definitive hosts, they shed L1 which develop in the intermediate hosts (snails and slugs) to the infective third stage (L3). The definitive hosts and humans may become infected ingesting raw or undercooked intermediate or paratenic hosts and vegetables contaminated with infected slug and snail mucus (Spratt, 2015).

Angiostrongylus costaricensis is reported in several countries of the Americas, from the United States to Argentina, with nearly 200 human cases described, most of which occurred in children (Graeff-Teixeira et al., 1991; Romero-Alegría et al., 2014). However, Morera and Amador (1998), on the basis of serological studies suggested that the number of human cases in Costa Rica could be up to 600 annually, also being the causative agent for $20 \%$ of acute abdominal conditions referred to the local National Children's Hospital (Abrahams-Sandi et al., 2011). Human infections with $A$. costaricensis have also been reported from Africa (Zaire) and Europe 
(France and Spain) (Graeff-Teixeira et al., 1991; Romero-Alegría et al., 2014).

White-nosed coati and raccoon in Costa Rica have expanded their range into both peri-urban and urban areas, and are considered as potential reservoirs of several zoonotic pathogens, such as Trypanosoma cruzi, Mycoplasma spp., Babesia spp., and Baylisascaris procyonis (Mehrkens et al., 2013; Baldi et al., 2016). Here we investigated the prevalence and features of $A$. costaricensis infection in white-nosed coatis and raccoons from Costa Rica to better understand their possible role in the epidemiology of this zoonotic infection.

\section{Materials and methods}

Fresh carcasses of 17 adult white-nosed coatis (ten females and seven males) and 44 raccoons ( 27 females and 17 males) were presented for necropsy to the Pathology Department of the Veterinary Medicine School of the Universidad Nacional (PSVMUN) in Heredia (Costa Rica). Carcasses were obtained from the North Pacific, Central Valley, and South Pacific areas of Costa Rica from February 2014 to March 2016. Fecal samples were processed by the Baermann technique for the detection of L1. Adult nematodes from mesenteric arteries and L1 from fecal samples were stored in $70 \%$ and $100 \%$ ethanol for morphological and molecular analysis, respectively. Retrospective cases from free-ranging white-nosed coatis and raccoons submitted as biopsy or necropsy material to the PSVMUN were also reviewed for a better appreciation of $A$. costaricensis or associated lesions.

\subsection{Molecular analyses}

The nematode identity was confirmed molecularly by specific amplification of cytochrome $c$ oxidase subunit 1 (cox1) and 18S rRNA gene fragments of adult worms according to previously described methodologies (Folmer et al., 1994; Patterson-Kane et al., 2009). Briefly, following genomic extraction using a commercial kit (DNeasy Blood \& Tissue Kit, Qiagen, GmbH, Hilden, Germany), $\operatorname{cox} 1(\sim 710 \mathrm{bp})$ and $18 \mathrm{~S}$ rRNA $(\sim 1700 \mathrm{bp})$ gene fragments were amplified and amplicons were resolved in (2\%) agarose gel, purified and sequenced directly in an automated sequencer (ABI-PRISM 377). Sequences were determined from both strands and compared with those available in GenBank ${ }^{\mathrm{TM}}$ dataset by Basic Local Alignment Search Tool (BLAST-http://blast.ncbi.nlm.nih.gov/Blast.cgi) (Altschul et al., 1997).

\section{Results and discussion}

Fourteen of 17 (82\%) fresh carcasses of white-nosed coatis were positive for $A$. costaricensis but no individual of raccoon was found infected. Infected carcasses were in moderate to poor body condition with variable amounts of abdominal transudate (from 10 to $35 \mathrm{ml}$ ), enlargement of mesenteric lymph nodes, marked dilation of mesenteric arteries associated with large granulomas ranging from 2 to $5 \mathrm{~cm}$ in diameter in association with adult nematodes (Figs. 1 and 2). A moderate to severe catarrhal enteritis was observed in all infected white-nosed coatis, mainly affecting the jejunum and ileum. The intestinal serosa was hyperemic with presence of fibrin. The liver, spleen and kidneys were congested in all cases.

At the histopathological exam, all infected cases showed a moderate to severe transmural enteritis with areas of epithelial erosion, blunting and atrophy of villi. The lamina propria was diffusely thickened by inflammatory infiltrate composed of lymphocytes, macrophages, and plasma cells. Several nematode larvae were observed in the mucosa, sub-mucosa and muscularis with presence

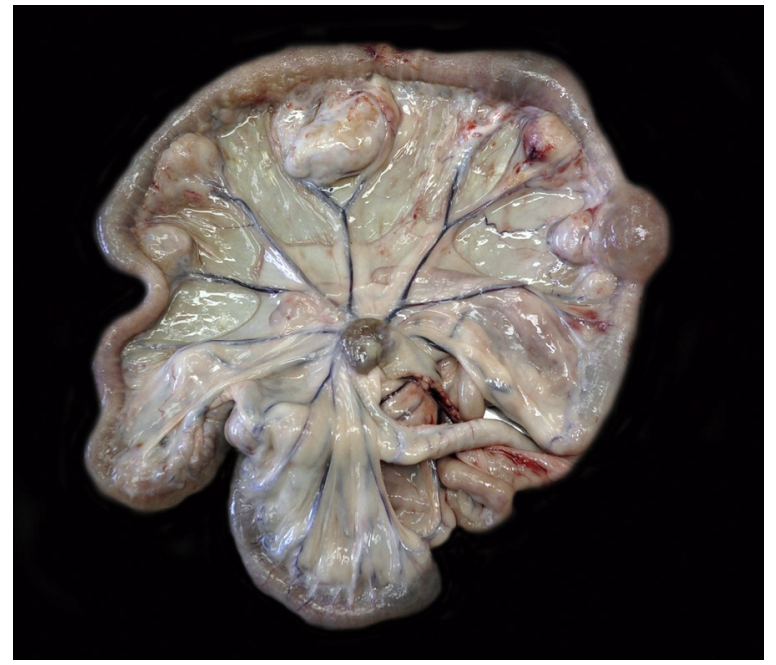

Fig. 1. Multiple large granulomas related to mesenteric arteries of the segments of the jejunum, ileum, caecum and proximal colon.

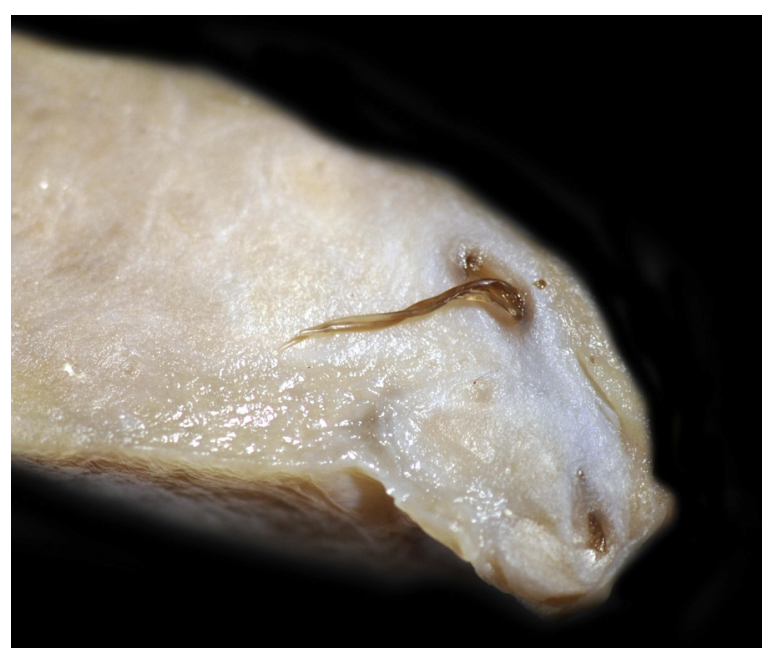

Fig. 2. Section of a large pyogranuloma associated with mesenteric arteries, note the adult individuals of $A$. costaricensis in the vascular lumen.

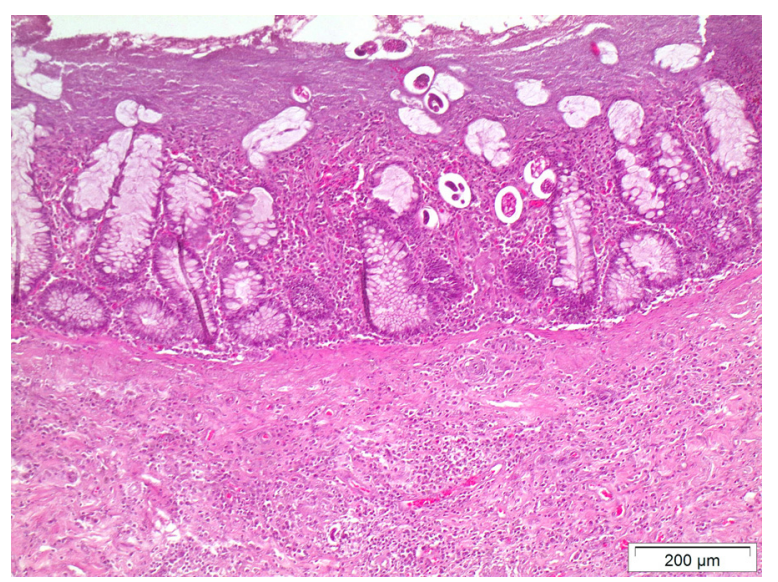

Fig. 3. Pyogranulomatous and eosinophilic ileitis with presence of $A$. costaricensis larvae in the mucosa. HE.

of granulomatous and eosinophilic inflammation (Fig. 3). Adult nematodes were also detected in the lumen of dilated mesenteric arteries, occluding partially or completely the arterial lumen 


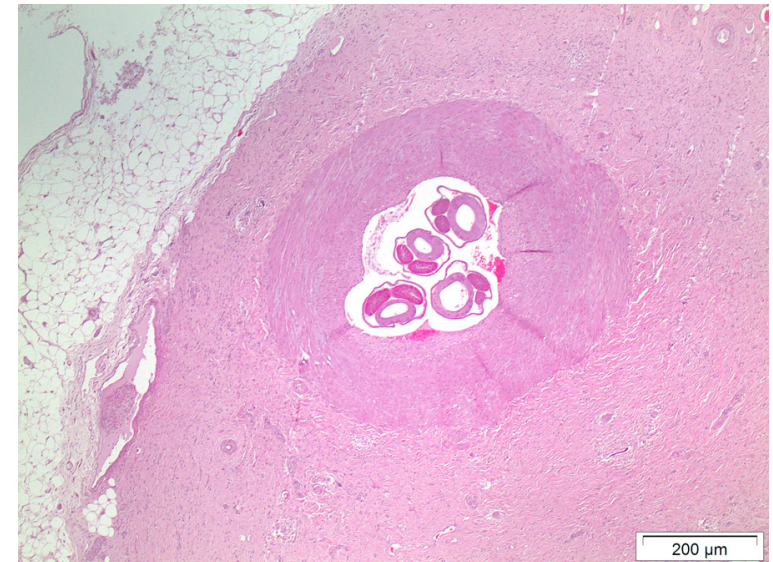

Fig. 4. Sections of adult $A$. costaricensis in the vascular lumen of the mesenteric artery; the artery is surround by a massive proliferation of granulation tissue and pyogranulomatous and eosinophilic inflammation. HE.

(Fig. 4). These worms were associated with severe pyogranulomatous and eosinophilic perivasculitis with the presence of epithelioid macrophages and multinucleated foreign body type giant cells with up to 28 nuclei. Mesenteric lymph nodes were hyperplasic in association with a severe pyogranulomatous and eosinophilic inflammation and the presence of several parasitic larvae. In a single case, extensive pyogranulomatous and eosinophilic confluent and bridging necrotizing hepatitis was observed associated with nematode larvae.

Adult worms and L1 obtained by Baermann technique were morphologically identified as A. costaricensis based on the species specific features described in Morera and Céspedes (1971), and Rebello et al. (2013). Histopathologically, the characteristics of nematode sections were compatible with those of $A$. costaricensis extracted from vessels as described in Graeff-Teixeira et al. (1991). The BLAST analysis of the cox 1 and 18S rRNA sequences produced here showed $99 \%$ and $100 \%$ homology, respectively, to those of $A$. costaricensis available in GenBank ${ }^{\mathrm{TM}}$ (Accession number: GQ398122 for cox1, LK942974 for 18S rRNA). Sequences were deposited in GenBank ${ }^{\mathrm{TM}}$ under accession number KX378964 for $18 \mathrm{~S}$ and $\mathrm{KX} 378965$ for $\operatorname{cox} 1$.

Retrospective cases obtained between July 2010 and December 2013 included 15 white-nosed coatis (eight males and six females) from South and North Pacific, and 53 raccoons (21 males and 32 females) from North Pacific, Central Valley, and South Pacific areas. Of all retrospective cases, only $4(27 \%)$ white-nosed coatis were found histopathologically infected with A. costaricensis (3 from South and one from North Pacific respectively).

The high prevalence of $A$. costaricensis suggested that this infection is enzootic among white-nosed coatis in Costa Rica, it being a naturally susceptible definitive host for the nematode in contrast to raccoons. L1 of an Angiostongylus species collected from feces of a white-nosed coati were found to experimentally develop in Biomphalaria glabrata snails (Monge et al., 1978). L3 larvae obtained 30 days later from those snails were orally inoculated into three cotton rats and, 22 days later, $\mathrm{L} 1$ were found in feces and adult nematodes of $A$. costaricensis were retrieved in mesen- teric arteries of the same animals. Aside from this experimental trial (Monge et al., 1978), no data have been published on freeranging wildlife reservoirs of $A$. costaricensis other than rodents (Spratt, 2015). Indeed, the report of $A$. costaricensis from mesenteric arteries of free-ranging raccoons and an opossum in Florida was never confirmed by fecal shedding of L1 (Miller et al., 2006). Our findings together with the consistent occurrence of populations of infected mollusk intermediate hosts in urban and peri-urban areas of Costa Rica with prevalence of infection $>50 \%$ for veronicellid slugs (Conejo and Morera, 1988), highlight the risk of abdominal angiostrongyliasis to humans and their pets, which, in turn, may act as reservoir hosts in the domestic environment (AlfaroAlarcón et al., 2016). Local residents should be informed about the risk of transmission and public health authorities should consider surveillance and control strategies to reduce intermediate host populations.

\section{References}

Abrahams-Sandi, E., Mesén-Ramírez, P., Suarez-Chacón, D., Fernández-Quezada, K., 2011. An indirect immunofluorescence antibody test employing whole eggs as the antigen for the diagnosis of abdominal angiostrongyliasis. Mem. Inst. Oswaldo Cruz. 106 (4), 390-393.

Alfaro-Alarcón, A., Veneziano, V., Galiero, G., Cerrone, A., Gutierrez, N., Chinchilla, A., Annoscia, G., Colella, V., Dantas-Torres, F., Otranto, D., Santoro, M., 2016. First report of a naturally patent infection of Angiostrongylus costaricensis in a dog. Vet. Parasitol. 212, 431-434.

Altschul, S.F., Madden, T.L., Schäffer, A.A., Zhang, J., Zhang, Z., Miller, W., Lipman, D.J., 1997. Gapped BLAST and PSI-BLAST: a new generation of protein database search programs. Nucleic Acids Res. 25, 3389-3402.

Baldi, M., Alvarado, G., Smith, S., Santoro, M., Bolaños, N., Jiménez, C., Hutter, S.E., Walzer, C., 2016. Baylisascaris procyonis parasites in raccoons, Costa Rica, 2014. Emerg. Infect. Dis. 22 (8), 1502-1503.

Conejo, M.E., Morera, P., 1988. Influence of the age of veronicellid slugs in Angiostrongylus costaricensis infection. Rev. Biol. Trop. 36 (2B), 519-526.

Folmer, O., Black, M., Hoeh, W., Lutz, R., Vrijenhoek, R., 1994. DNA primers for amplification of mitochondrial cytochrome c oxidase subunit I from diverse metazoan invertebrates. Mol. Mar. Biol. Biotechnol. 3, 294-299.

Graeff-Teixeira, C., Camillo-Coura, L., Lenzi, H.L., 1991. Histopathological criteria for the diagnosis of abdominal angiostrongyliasis. Parasitol. Res. 77, 606-611.

Mehrkens, L.R., Shender, L.A., Yabsley, M.J., Shock, B.C., Chinchilla, F.A., Suarez, J., Gilardi, K.V.K., 2013. White nosed coatis (Nasua narica) are a potential reservoir of Trypanosoma cruzi and other potentially zoonotic pathogens in Monteverde, Costa Rica. J. Wildl. Dis. 49 (4), 1014-1018.

Miller, C.L., Kinsella, J.M., Garner, M.M., Evans, S., Gullett, P.A., Schmidt, R.E., 2006. Endemic infections of Parastrongylus (=Angiostrongylus) costaricensis in two species of nonhuman primates, raccoons, and an opossum from Miami, Florida. J. Parasitol. 92 (2), 406-408.

Monge, E., Arroyo, R., Solano, E., 1978. A new definitive natural host of Angiostrongylus costaricensis (Morera and Cespedes 1971). J. Parasitol. 64, 34.

Morera, P., Amador, J., 1998. Prevalencia de la angiostrongilosis abdominal y la distribución estacional de la precipitación. Rev. Costarric. Salud Pública. 7 (13), $1-14$.

Morera, P., Céspedes, R., 1971. Angiostrongylus costaricensis n. sp. (Nematoda Metastrongyloidea): a new lungworm occurring in man in Costa Rica. Rev. Biol. Trop. 18, 173-185.

Patterson-Kane, J.C., Gibbons, L.M., Jefferies, R., Morgan, E.R., Wenzlow, N., Redrobe, S.P., 2009. Pneumonia from Angiostrongylus vasorum infection in a red panda (Ailurus fulgens fulgens). J. Vet. Diagn. Invest. 21, 270-273.

Rebello, K.M., Menna-Barreto, R.F.S., Chagas-Moutinho, V.A., Mota, E.M., Perales, J., Neves-Ferreira, A.G.C., Oliveira-Menezes, A., Lenzi, H., 2013. Morphological aspects of Angiostrongylus costaricensis by light and scanning electron microscopy. Acta Trop. 127, 191-198.

Romero-Alegría, A., Belhassen-García, M., Velasco-Tirado, V., Garcia-Mingo, A., Alvela-Suárez, L., Pardo-Lledias, J., Cordero Sánchez, M., 2014. Angiostrongylus costaricensis: systematic review of case reports. Adv. Infect. Dis, 4, 36-41.

Spratt, D.M., 2015. Species of Angiostrongylus (Nematoda: Metastrongyloidea) in wildlife: a review. Int. J. Parasitol. Parasites Wildl. 4, 178-189. 